\section{BMJ Open} Ophthalmology

\title{
Prospective pilot study comparing deep sclerectomy outcomes with a long-term and intense corticosteroid treatment
} versus a standard one

\author{
Aitor Lanzagorta-Aresti, Marta Perez-Lopez, Juan Maria Davo-Cabrera, \\ Elena Palacios-Pozo
}

To cite: Lanzagorta-Aresti A, Perez-Lopez M, Davo-Cabrera JM, et al. Prospective pilot study comparing deep sclerectomy outcomes with a long-term and intense corticosteroid treatment versus a standard one. BMJ Open Ophthalmology 2018;3:e000165. doi:10.1136/ bmjophth-2018-000165

Received 25 March 2018 Revised 13 September 2018 Accepted 24 September 2018

\section{Check for updates}

(C) Author(s) (or their employer(s)) 2018. Re-use permitted under CC BY-NC. No commercial re-use. See rights and permissions. Published by BMJ.

Glaucoma and NeuroOphthalmology Department, FISABIO Oftalmologia, Valencia, Spain

Correspondence to Aitor Lanzagorta-Aresti; aitorlanzagorta@hotmail.com

\section{ABSTRACT}

Objective To compare prospectively intraocular pressure (IOP) results after deep sclerectomy (DS) using a topical short-term corticosteroid treatment (STCT, 1 month) versus a topical long-term and intense corticosteroid treatment (LTCT, 6 months) in a two2 year-follow-up.

Methods Patients with medically uncontrolled open angle glaucoma were prospectively recruited and underwent a DS.

Results We operated 45 eyes of 45 patients, 22 in STCT group and 23 in LTCT group. Median preoperative IOP was 27 (22-36.75) $\mathrm{mm} \mathrm{Hg}$ for STCT and for 25 (22-28) $\mathrm{mm}$ $\mathrm{Hg}$ for LTCT group without significant difference $(p=0.195)$. Median postoperative IOP was 4 (3-6.25) $\mathrm{mm} \mathrm{Hg}$ in STCT group versus $2(0-5) \mathrm{mm} \mathrm{Hg}$ in LTCT at day $1(\mathrm{p}=0.003)$; 8.5 (5.75-11.25) mm Hg (STCT) vs 6 (4-9) mm Hg (LTCT) at week $1(p=0.079) ; 17.5(14.75-22.25) \mathrm{mm} \mathrm{Hg}$ (STCT) vs $13(10-14) \mathrm{mm} \mathrm{Hg}$ (LTCT) at month $1(p=0.001) ; 16$ (12-20) $\mathrm{mm} \mathrm{Hg}$ (STCT) vs 12 (10-15) $\mathrm{mm} \mathrm{Hg}$ (LTCT) at month 3 ( $p=0.008) ; 17$ (14-20) mm Hg (STCT) vs 12 (10-14) $\mathrm{mm} \mathrm{Hg}$ (LTCT) at month $6(\mathrm{p}=0.000) ; 16$ (14-20) $\mathrm{mm} \mathrm{Hg}$ (STCT) vs 14 (10-16) $\mathrm{mm} \mathrm{Hg}$ (LTCT) at year 1 $(p=0.002)$ and $17.5(15-19) \mathrm{mm} \mathrm{Hg}$ (STCT) vs $14(12-16)$ $\mathrm{mm} \mathrm{Hg}(\mathrm{LTCT})$ at year $2(\mathrm{p}=0.001)$. The complete success rate was $54.5 \%$ in STCT and $87 \%$ in LTCT ( $p=0.018)$. Conclusions A long-term and intensive postoperative treatment enhances success rate in DS compared with a standard protocol.

\section{OBJECTIVE}

Several procedures have been described to modulate wound healing and improve glaucoma surgery outcomes. Avoiding hypotensive eyedrops that contain preservatives before surgery, meticulous surgical technique and the use of antifibrotic agents such as mitomycin C (MMC) or 5-fluorouracil (5-FU) have been previously reported. Postoperative glucocorticoid drops are frequently used to blunt the healing process. They have been proven to be effective in decreasing the inflammatory response that can lead to the bleb failure. ${ }^{1}$

\section{Key messages}

What is already known about this subject?

Bleb fibrosis is the main cause of glaucoma surgery failure.

\section{What are the new findings?}

The new finding is that a long and intensive corticoid treatment after glaucoma surgery improves outcomes.

How might these results change the focus of research or clinical practice?

It can change the standard postoperative treatment in glaucoma.

Although corticosteroids seem to improve glaucoma surgery outcomes, there are no prospective studies to elucidate the most effective route, dosage and duration of postoperative treatment in glaucoma surgery.

Deep sclerectomy (DS) is a non-penetrating filtration procedure for glaucoma surgical treatment of glaucoma that was designed to avoid usual complications related to trabeculectomy without compromising the success rate such as postoperative hypotony. Most accepted postoperative treatment is a combination of topical antibiotic and corticosteroid drops three times a day for 4 weeks. ${ }^{23}$

Recently, anterior segment optical coherence tomography has been used to study scleral lake morphology and to detect its changes of the scleral lake after DS in a longterm follow-up. It has been suggested that there are prospective changes related to wound healing process that can extend more than 6 months $^{4}$ postoperatively. According to these findings, it would be reasonable to extend anti-inflammatory treatment to at least 6 months, while conjunctival bleb is still remodelling. Although new approaches to develop implants with a sustained release of 
corticosteroids have been investigated, they are not available yet as far as we are aware.

The aim of the present study is to evaluate the DS surgery outcomes comparing a postoperative long-term and intensive topical corticosteroid treatment (LTCT, 6 months) versus short-term and standard topical corticosteroid treatment (STCT, 4 weeks).

\section{MATERIAL AND METHODS}

We performed a prospective, consecutive randomised study of 51 eyes of 51 patients with medically uncontrolled open angle glaucoma between January 2012 and November 2014. This study was approved by the Review Board of FISABIO and conformed to the Declaration of Helsinki. Informed written consent was obtained from all subjects.

Patients were included if they had uncontrolled primary open angle glaucoma (defined as an IOP $>21$ mm Hg measured with a Goldmann applanation tonometer besides maximal tolerable medical treatment or well documented progression of visual field (VF) or nerve fibre layer defects), central corneal thickness between 520 and $580 \mu \mathrm{m}$ and grade III or IV in Schaffer classification in gonioscopy. All the patients were Caucasian and phakic.

Exclusion criteria were defined as previous surgery, advanced lens opacities (more than grade 3 for nuclear opalescence, nuclear colour and cortical changes and more than 2 for subcapsular posterior changes in the Lens Opacities Classification System III), laser trabeculoplasty and iridocorneal angle abnormalities or grade $\leq$ II in Schaffer classification.

Preoperatively, patients underwent a complete ophthalmic examination including best-corrected visual acuity (BCVA) assessment, Goldmann applanation tonometry (GAT) and gonioscopy.

All measurements were repeated in visits performed 1 day, 1 week, 1 month, 3 months, 6 months, 1 year and 2 years after surgery. VF was performed in the preoperative visit and in the visits 6 months, 1 year and 2 years after surgery and mean defect value was collected.

Two individuals, one operating the slit-lamp and the dial and another one reading the dial and recording the results performed GAT twice. If the two measurements differ more than $4 \mathrm{mmHg}$ a third measurement was obtained. The definitive IOP was the average among the two or three measurements.

All the patients were operated by the same surgeon (ALA) who performed DS surgery as the preferable technique used for open angle glaucoma in our Institution. The surgical technique consisted in dissecting a one-third scleral thickness flap measuring $5 \times 5 \mathrm{~mm}$ and extending it $2 \mathrm{~mm}$ in clear cornea. After, we put on $2 \mathrm{~min}$ of MMC $0.2 \%$ with sponges under conjunctiva and superficial flap. We created a triangular deep scleral flap that was posteriorly removed, necessary to reach Schlemm's canal. A retina surgery 25G micro-forceps was used to unroof it. After checking aqueous humour was percolating through the remaining trabeculo-Descemet's membrane (TMD), an incision until suprachoroidal space was created and the implant was placed in this pocket without suturing it. The implant used was T-flux (Carl Zeiss Meditec, Dublin, California, USA), a non-absorbable biocompatible acrylic device. The superficial flap was repositioned and no sutures were used to close it. Two single 8-0-Nylon sutures were used to seal conjunctiva.

Postoperatively treatment protocol (STCT and LTCT) was randomly assigned to each patient using online software (http://www.randomization.com).

STCT postoperative treatment was moxifloxacin three times a day for a week and dexamethasone four times a day for 4 weeks.

LTCT postoperative treatment was moxifloxacin three times a day for a week and dexamethasone every 2 hours for a month, every 4 hours during the second month, every 6 hours during the third month, every 8 hours during the fourth month, every 12 hours during the fifth month and once a day during the last month (6 months).

Surgery complete success was calculated for IOP $<21$ $\mathrm{mm} \mathrm{Hg},<18 \mathrm{~mm} \mathrm{Hg}$ and $<15 \mathrm{~mm} \mathrm{Hg}$ without glaucoma medication and a qualified success was defined as IOP was $<21 \mathrm{~mm} \mathrm{Hg}$ with or without glaucoma medication. Failure was considered when IOP was greater than 21 $\mathrm{mm} \mathrm{Hg}$ with or without medication or further glaucoma surgery was necessary. Goniopuncture with Nd:YAG or subconjunctival injection with MMC $0.02 \%$ to achieve an IOP $\leq 21 \mathrm{~mm} \mathrm{Hg}$ was considered a qualified success. If a failure was documented, a new surgery with Ahmed valve was performed.

\section{Data analysis}

Data were analysed using SPSS V.21.0 software (SPSS, Chicago, Illinois, USA). Given the small sample size in each group, the non-parametric tests were used. The median values and IQR (P25-P75) were calculated for quantitative value of study variables before and after surgery. Wilcoxon signed-rank test was used to paired samples to compare differences before and after treatment, Mann-Whitney $\mathrm{U}$ test was used to compare the data between both groups ( 1 month and 6 months treatment). Kaplan-Meier curves for long-term success rate analysis were compared using log rank test. $\mathrm{P}<0.05$ was considered significant.

\section{RESULTS}

Fifty-one patients satisfied the inclusion criteria and were initially recruited, three were excluded from the study because a perforation in the TMD membrane occurred during the surgery, one of them suffered from a spontaneous break of the TMD postoperatively and two of them did not attend to follow-up visits (six patients dropped out the study in total). Of the 45 patients (22 patients in STCT group and 23 patients in LTCT group), 34.8\% were men and $65.2 \%$ were women. No significant difference in sex distribution between both groups was found $(\mathrm{p}=0.579)$. Demographic distribution is shown in table 1. 
Table 1 Preoperative demographic distribution of STCT and LTCT

\begin{tabular}{|c|c|c|c|c|c|}
\hline & \multicolumn{2}{|l|}{ STCT } & \multicolumn{2}{|l|}{ LTCT } & \multirow[b]{2}{*}{ P values } \\
\hline & Median & Range & Median & Range & \\
\hline Age & 66 & $60.25-74.5$ & 73 & $60-77$ & 0.254 \\
\hline Number medications & 3 & $3-4$ & 3 & $3-4$ & 1 \\
\hline
\end{tabular}

$\mathrm{P}<0.005$, Wilcoxon test.

LTCT, long-term corticosteroid treatment; STCT, short term corticosteroid treatment.

Median preoperative and postoperative IOP values in STCT group and LTCT group are shown in table 2.

A significant reduction of IOP in STCT and LTCT $(\mathrm{p}<0.05)$ was found at last visit (2 years follow-up). Complete success rate was $54.5 \%$ in STCT and $87 \%$ in LTCT ( $p=0.018$ ) for IOP $<21 \mathrm{~mm} \mathrm{Hg}$. Long-term complete success probabilities using a Kaplan-Meier survival curve are shown in figure 1 . For $18 \mathrm{~mm} \mathrm{Hg}$ (figure 2), success rate was $40.9 \%$ in STCT and $78.3 \%$ in LTCT $(\mathrm{p}=0.019)$. For $16 \mathrm{mmHg}$ (figure 3 ), success rate was $31.8 \%$ in STCT and $65.2 \%(\mathrm{p}=0.021)$

Qualified success for IOP $<21 \mathrm{~mm} \mathrm{Hg}$ was $90.9 \%$ in STCT and $100 \%$ in LTCT $(\mathrm{p}=0.13)$. The median number of medications was reduced from $3(3-4)$ to $0(0-2)$ in the STCT group $(\mathrm{p}<0.05)$ and from $3(3-4))$ to $0(0-0)$ in the LTCT group $(\mathrm{p}<0.05)$. The difference in the number of medications between both groups after 2 years of follow-up was significant ( $\mathrm{p}=0.026$ ).

Goniopuncture with the Nd:YAG laser was performed on five patients $(22.7 \%)$ in LTCT and three patients in STCT $(13.0 \%)$ and the difference was not significant $(\mathrm{p}=0.477)$. In STCT group, two subconjunctival injections with MMC (9.1\%) and two new surgeries with Ahmed valve implant $(9.1 \%)$ were performed. If we compare overall procedures needed between both groups, no significant difference was observed $(p=0.124)$. No subconjunctival injections or extra surgery was performed in LTCT.

No clinically significant cataract development was observed in either group. In LTCT group, preoperative median BCVA was $0.1(0.0-0.2)$ and 2 years after surgery was $0.1(0.0-0-15)$ without significant change $(p=0.5)$. In
STCT, preoperative mean BCVA was $0.1(0.0-0.15)$ and after surgery was $0.1(0.0-0.15)$ without significant difference $(p=0.7)$. There were no significant differences in median BCVA after surgery between both groups $(\mathrm{p}=0.5)$.

When we compare VFs, in LTCT group previous median MD was $-10.3(6.35-15.9) \mathrm{dB}$ and 2 years after surgery it was $-11.95(5.95-16.25) \mathrm{dB}(\mathrm{p}=0.7)$. On the other hand, in STCT group preoperative median MD was 10.35 (5.85-17.3) dB and postoperatively was 10.95 (5.15-16.65) dB ( $\mathrm{p}=0.8)$.

We did not report any case of shallow anterior chamber or choroidal detachment in either group. Only five cases (three in STCT arm and two in LTCT arm) presented a mild hyphema at the day after surgery but this complication was solved in less than 1 week without further manoeuvres.

\section{DISCUSSION}

This study demonstrates the efficacy of a long-term and intensive topical corticosteroid treatment to improve DS outcome. As far as we know, this is the first prospective study that compares two different postoperative treatment dosages of topical corticosteroids for DS in terms of efficacy. In this study, a long-term and intensive therapy with topical corticosteroids postoperatively led to a greater and more sustained IOP decrease after DS.

It is important to point out there were no significant differences in median IOP at 1 week postoperative visit, whereas there was a statistical significance from 1-monthvisit until 2-year-visit. This result suggests an influence of postoperative treatment. The number of medications was

Table 2 Comparative IOP results of STCT and LTCT

\begin{tabular}{|c|c|c|c|c|c|}
\hline \multirow[b]{2}{*}{ Time } & \multicolumn{2}{|c|}{ IOP STCT (mm Hg) } & \multicolumn{2}{|c|}{ IOP LTCT $(\mathrm{mm} \mathrm{Hg})$} & \multirow[b]{2}{*}{$P$ values } \\
\hline & Median & Range & Median & Range & \\
\hline Preoperative & 27 & $22-36.75$ & 25 & $22-28$ & 0.195 \\
\hline 1 day & 4 & $3-6.25$ & 2 & $0-5$ & $0.003^{*}$ \\
\hline 1 week & 8.5 & $5.75-11.25$ & 6 & $4-9$ & 0.079 \\
\hline 1 month & 17.5 & $14.75-22.25$ & 13 & $10-14$ & $0.001^{\star}$ \\
\hline 3 months & 16 & $12-20$ & 12 & $10-15$ & $0.008^{*}$ \\
\hline 6 months & 17 & $14-20$ & 12 & $10-14$ & $0.000^{\star}$ \\
\hline 1 year & 16 & $14-20$ & 14 & $10-16$ & $0.002^{*}$ \\
\hline 2 years & 17.5 & $15-19$ & 14 & $12-16$ & $0.001^{\star}$ \\
\hline
\end{tabular}

$\mathrm{P}<0.005$, Wilcoxon test.

IOP, intraocular pressure; LTCT, long-term corticosteroid treatment; STCT, short-term corticosteroid treatment. 


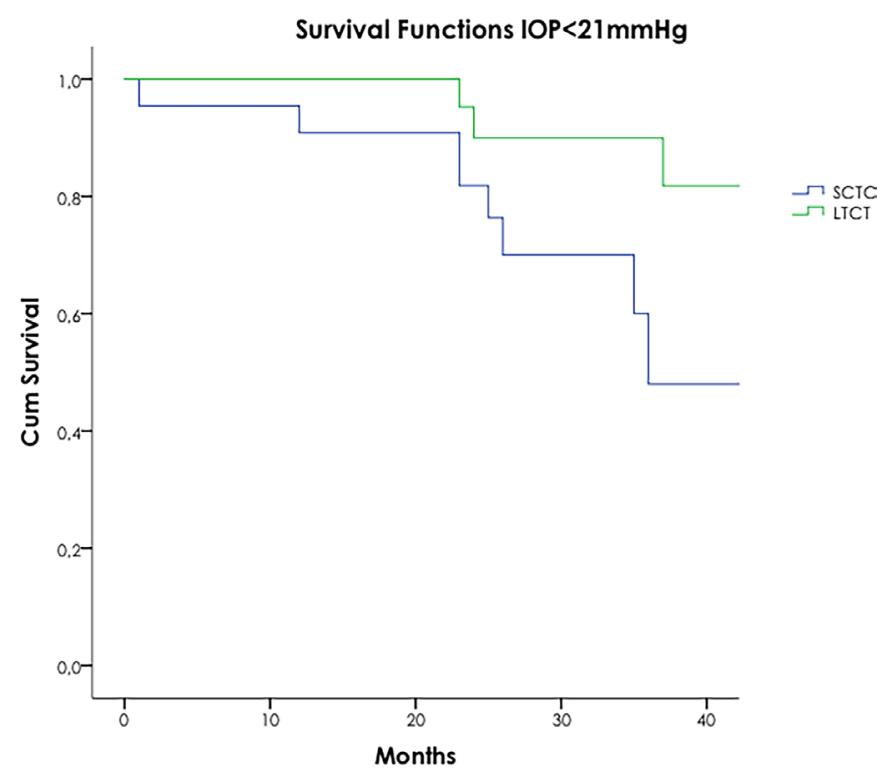

Figure 1 Long-term cumulative complete success probability using Kaplan-Meier life-table analysis for IOP $<21$ $\mathrm{mm} \mathrm{Hg}, 18 \mathrm{~mm} \mathrm{Hg}$ and $16 \mathrm{~mm} \mathrm{Hg}$. IOP, intraocular pressure; LTCT, long-term corticoid treatment; STCT, short-term corticoid treatment.

also reduced in both groups to 0 although it is statistically significant because we are using median value and not the mean one. There was not difference between extra procedures required to decrease IOP comparing both treatment groups. However, LTCT needed more goniopuncture (considered by many authors as a normal step in the postoperative period) whereas in STCT group 4 rescue surgeries were needed. Therefore, although the

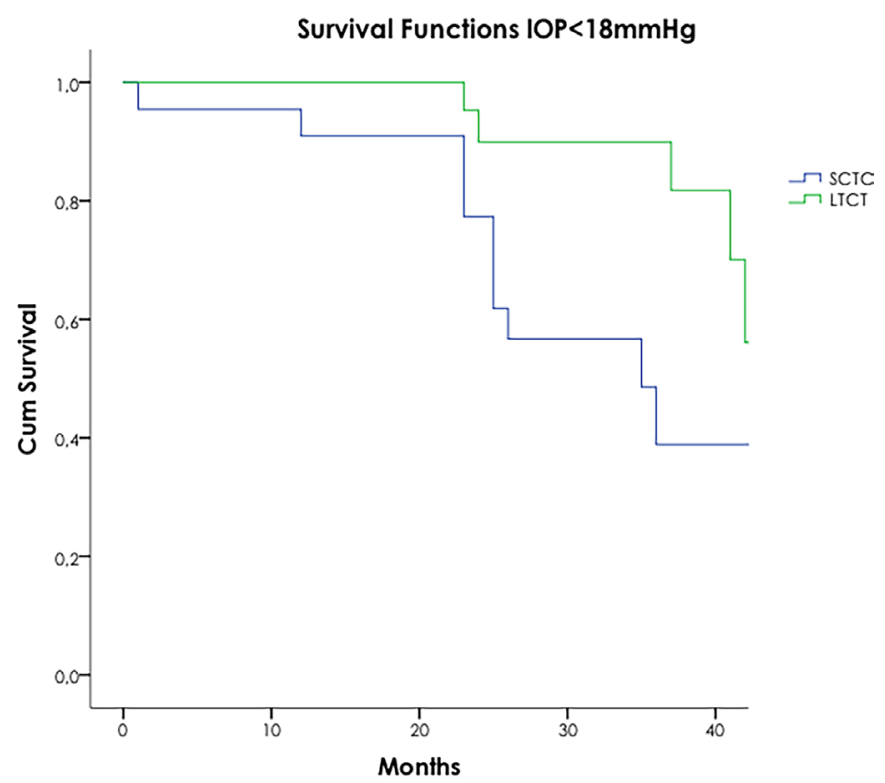

Figure 2 Long-term cumulative complete success probability using Kaplan-Meier life-table analysis for IOP $<21$ $\mathrm{mm} \mathrm{Hg}, 18 \mathrm{~mm} \mathrm{Hg}$ and $16 \mathrm{~mm} \mathrm{Hg}$. IOP, intraocular pressure; LTCT, long-term corticoid treatment; STCT, short-term corticoid treatment.

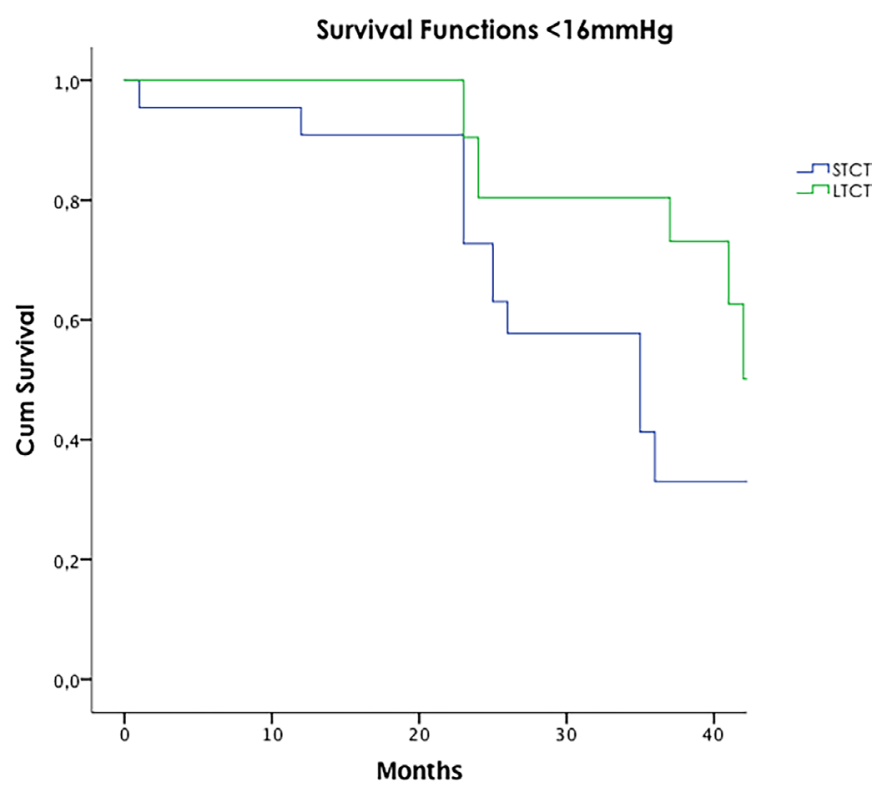

Figure 3 Long-term cumulative complete success probability using Kaplan-Meier life-table analysis for IOP $<21$ $\mathrm{mm} \mathrm{Hg}, 18 \mathrm{~mm} \mathrm{Hg}$ and $16 \mathrm{~mm} \mathrm{Hg}$. IOP, intraocular pressure; LTCT, long-term corticoid treatment; STCT, short-term corticoid treatment.

amount of procedures in both groups is similar, the quality of them points out a greater failure rate in STCT.

Cheng et al performed a systematic review of the efficacy of several non-penetrating glaucoma surgical procedures, including DS. Twenty-nine randomised clinical trials were included in the meta-analysis trials and 1455 patients were evaluated, eight arms reporting DS. IOP reduction at 24 months was $35.2 \%$ (CI 95\% 30.0\% to $40.4 \%$ ) and complete success rate was $44 \%$ (CI $18.9 \%$ to $72.5 \%)$. The qualified success rate at the endpoint was $71.7 \%$ for the DS. Moreover, they observed a gradual loss of IOP-lowering effect over time. ${ }^{5}$ In our study, the complete success rate in STCT group at 24 months was $54.5 \%$, which is comparable to previously published data. However, complete success rate in LTCT group was $87 \%$ at 24 months follow-up which is higher than those reported to date. Therefore our study suggests a beneficial effect of postoperatively long-term topical corticosteroids treatment in improving DS outcome.

The use of either goniopuncture or glaucoma medication increased the success rate, but it is difficult to compare the results because many studies include additional interventions as normal practice to get success, introducing a bias. In this meta-analysis, the success rate of the trabeculectomy at 2 years was $62.1 \%$ (CI $49.4 \%$ to $73.4 \%$ ) and the qualified success at the endpoint was $90.8 \%$ (CI $85.4 \%$ to $95.4 \%$ ).

The beneficial effect of corticosteroids is due to a well-known action in the inflammatory cascade and also related to a more theoretical mechanism where the decreased outflow in the trabecular meshwork (TM) leads to an increased outflow to the created scleral lake. 
Glaucoma filtering surgery failure is usually due to excessive wound healing under the conjunctiva.

Four phases have been described in the healing process: coagulative, inflammatory, proliferative and remodelling. In the coagulative phase, the blood cells and platelets from the incised vasculature release prostaglandins, leukotrienes, histamine and serotonin. Platelets also release Platelet derived growth factor (PDGF), vascular endothelial growth factor (VEGF) and insulin-like growth factor. In the inflammatory phase, monocytes differentiate into tissue macrophagues. These release PDGF, fibroblast growth factor and transforming growth factor- $\beta$. The fibroblasts are the main cell involved in proliferative phase. They transform into myofibroblasts, a more contractile phenotype. Extracellular matrix degradation is mediated by metallo-proteinases via the removal of hyaluronan and fibronectin from the tissue leading to apoptosis of fibroblasts, main event of remodelling phase. ${ }^{67}$

The key of success in glaucoma surgery involves an optimal balance between conjunctival healing and scar formation in the filtering bleb. Several approaches have been suggested to achieve this delicate goal.

Most of the efforts to reduce glaucoma surgery failure are based on the development of antifibrotic agents. These antimetabolites (MMC and 5-FU) have contributed to better outcomes in terms of postoperative IOP control but their use have also increased the complication rate of filtration surgery. One of the most undesirable side effects is the late-onset leak related to bleb ischaemia. This leak can lead to a severe hypotony maculopathy, shallowing of the anterior chamber, cataract formation, corneal decompensation, choroidal effusion, blebitis and endophthalmitis. ${ }^{1}$ In order to decrease these complications, the Moorfield's safer surgery was developed using a fornix-based conjunctival incision and a larger antimetabolite treatment area. Newer techniques in delivering antimetabolites are being investigating.

Other forms to modulate wound healing with more acceptable side effects have been explored. As we mentioned before, VEGF plays an important role in angiogenesis and its relation with the healing process in filtering bleb has been also investigated. ${ }^{8}$ Lopilly Park $e t$ al found a significant association between IOP and VEGF level in the Tenon's tissue. ${ }^{9}$ So, a VEGF inhibition in the bleb would improve surgery outcomes. However, no conclusive evidence has been found in the efficacy and in dosage or method of delivery in several studies. ${ }^{10-17}$

Two randomised clinical trials evaluated the benefit of subconjunctival hyaluronate. Lopes et al compared hyaluronate versus balance salt solution without finding significant differences after a mean follow-up of 12 months. ${ }^{18}$ Naravanaswamy did not observe differences comparing hyaluronate mixed 5-FU versus 5-FU alone either. $^{19}$

Glucocorticoids modify the inflammatory process at several stages of the cascade of inflammation including reduction in concentration, migration and activation of leucocytes, inhibition of activity of macrophagues, mitigation the reaction of immune system to presented antigens and reduction in vascular permeability. ${ }^{6}$

In spite of the action in nearly all the levels of inflammatory response, corticosteroids have been considered a rudimentary form of scarring suppressor. No many studies have been performed in order to study the efficacy and optimal dosage.

Corticosteroids must be used after any filtering surgical procedure. However, this treatment can cause a rise in IOP in a percentage of subjects, especially those suffering from glaucoma and this is one of the most concerns with an intensive and long use. This has been described before in previous studies, where corticosteroids induced an increase of IOP response in both normal and glaucomatous eyes, patients with glaucoma being more susceptible. In open-angle glaucoma, $46 \%-92 \%$ of patients may develop a steroid-induced IOP rise whereas the prevalence decreases to $17 \%-36 \%$ in operated patients with glaucoma. ${ }^{20}$ Steroid-induced ocular hypertension is associated with specific changes in the TM, such as an increased deposition of extracellular matrix and an altered TM cell function. Although this IOP increase can happen in operated eyes, the proportion is considerably less important.

The most accepted explanation is that in operated eyes a great amount of aqueous humour bypasses the TM through the ostium in trabeculectomy or the TMD in DS.

However, in our study, despite intensive topical corticosteroid treatment, any of the patients developed an IOP rise postoperatively. The intensified corticosteroid therapy after glaucoma surgery is not new. A 10-year follow-up of a prospective randomised trial of postoperative corticosteroids reported that an intensified therapy in the first 4 weeks was beneficial to stabilise IOP after trabeculectomy. ${ }^{21}$ In the most extended postoperative treatment after trabeculectomy, the regimen used is two hourly prednisolone acetate or dexamethasone drops during waking hours for the first month before slowly tapering the frequency over the following months. ${ }^{22}$ However, this kind of regimen had not extended to DS because a more educed inflammation after DS than in trabeculectomy and the absence of iridectomy. Our study shows this treatment is also useful in DS.

It has been suggested that the trabecular pathway outflow is reduced after a successful trabeculectomy. In fact, the diameter of Schlemm's canal decreases after filtration surgery because of a probable underperfusion of the TM. ${ }^{723}$ On the other hand, although the mechanism of glucocorticoid-induced outflow obstruction is not very well known, it has been associated with the accumulation of extracellular matrix material, particularly in the juxtacanalicular tissue. ${ }^{25}$

The most important study limitation is the small sample size. Nevertheless, the difference between the two treatment regimens was so significant in the preliminary results in favour of long-term and intensive treatment that 6-month treatment was established as postoperative 
protocol for DS surgeries. Therefore, recruitment of patients in the STCT group was discontinued.

In conclusion, we demonstrated that a long-term and intensive topical corticosteroid postoperative treatment enhances success rate in DS compared with the standard protocol. A greater IOP reduction, lower need of postoperative medications and further surgeries and a longer efficacy were achieved in the LTCT compared with STCT. No complications associated with this regimen were found.

Contributors AL-A planned the study, performed surgery and submitted the study. MP-L made statistics. MP-L, EP-P and JMD-C collected data and reviewed the manuscript.

Funding The authors have not declared a specific grant for this research from any funding agency in the public, commercial or not-for-profit sectors.

Competing interests None declared.

Patient consent Not required.

Ethics approval FOM Institutional Board.

Provenance and peer review Not commissioned; externally peer reviewed.

Open access This is an open access article distributed in accordance with the Creative Commons Attribution Non Commercial (CC BY-NC 4.0) license, which permits others to distribute, remix, adapt, build upon this work non-commercially, and license their derivative works on different terms, provided the original work is properly cited, appropriate credit is given, any changes made indicated, and the use is non-commercial. See: http://creativecommons.org/licenses/by-nc/4.0/

\section{REFERENCES}

1. Fan Gaskin JC, Nguyen DQ, Soon Ang G, et al. Wound healing modulation in glaucoma filtration surgery-conventional practices and new perspectives: the role of antifibrotic agents (Part I). J Curr Glaucoma Pract 2014;8:37-45.

2. Shaarawy TM, Sherwood MB, Crowston JG. Glaucoma. 2nd. Philadelphia: Elsevier Health Sciences, 2015.

3. Shaarawy T, Nguyen C, Schnyder C, et al. Comparative study between deep sclerectomy with and without collagen implant: long term follow up. Br J Ophthalmol 2004;88:95-8.

4. Urcola JA, Dalmasso C, Cabrerizo J. Prospective morphological subconjunctive analysis by spectral domain optical coherence tomography of blebless glaucoma surgeries. Invest Ophthalmol Vis Sci 2013;54:4760.

5. Cheng JW, Cheng SW, Cai JP, et al. Systematic overview of the efficacy of nonpenetrating glaucoma surgery in the treatment of open angle glaucoma. Med Sci Monit 2011;17:RA155-63.

6. Masoumpour MB, Nowroozzadeh MH, Razeghinejad MR. Current and future techniques in wound healing modulation after glaucoma filtering surgeries. Open Ophthalmol J 2016;10:68-85.
7. Van Bergen T, Van de Velde S, Vandewalle E, et al. Improving patient outcomes following glaucoma surgery: state of the art and future perspectives. Clin Ophthalmol 2014;8:857-11.

8. Fan Gaskin JC, Nguyen DQ, Soon Ang G, et al. Wound healing modulation in glaucoma filtration surgery- conventional practices and new perspectives: antivascular endothelial growth factor and novel agents (Part II). J Curr Glaucoma Pract 2014;8:46-53.

9. Lopilly Park HY, Kim JH, Ahn MD, et al. Level of vascular endothelial growth factor in tenon tissue and results of glaucoma surgery. Arch Ophthalmol 2012;130:685-9.

10. Sengupta S, Venkatesh R, Ravindran RD. Safety and efficacy of using off-label bevacizumab versus mitomycin $\mathrm{C}$ to prevent bleb failure in a single-site phacotrabeculectomy by a randomized controlled clinical trial. J Glaucoma 2012;21:450-9.

11. Tai TY, Moster MR, Pro MJ, et al. Needle bleb revision with bevacizumab and mitomycin $\mathrm{C}$ compared with mitomycin $\mathrm{C}$ alone for failing filtration blebs. J Glaucoma 2015;24:311-5.

12. Nilforushan N, Yadgari M, Kish SK, et al. Subconjunctival bevacizumab versus mitomycin $\mathrm{C}$ adjunctive to trabeculectomy. Am J Ophthalmol 2012;153:352-7.

13. Jurkowska-Dudzińska J, Kosior-Jarecka E, Zarnowski T. Comparison of the use of 5-fluorouracil and bevacizumab in primary trabeculectomy: results at 1 year. Clin Exp Ophthalmol 2012;40:e135-42.

14. Coote MA, Ruddle JB, Qin Q, et al. Vascular changes after intra-bleb injection of bevacizumab. J Glaucoma 2008;17:517-8.

15. Ozgonul C, Mumcuoglu T, Gunal A. The effect of bevacizumab on wound healing modulation in an experimental trabeculectomy model. Curr Eye Res 2014;39:451-9.

16. Simsek T, Cankaya AB, Elgin U. Comparison of needle revision with subconjunctival bevacizumab and 5 -fluorouracil injection of failed trabeculectomy blebs. J Ocul Pharmacol Ther 2012;28:542-6.

17. O'Neill EC, Qin Q, Van Bergen NJ, et al. Antifibrotic activity of bevacizumab on human Tenon's fibroblasts in vitro. Invest Ophthalmol Vis Sci 2010;51:6524-32.

18. Lopes JF, Moster MR, Wilson RP, et al. Subconjunctival sodium hyaluronate $2.3 \%$ in trabeculectomy: a prospective randomized clinical trial. Ophthalmology 2006;113:756-60.

19. Narayanaswamy AK, Lee K, Zhen M, et al. Randomized, controlled trial of a sustained delivery formulation of 5-fluorouracil for the treatment of failing blebs. Ophthalmology 2012;119:314-20.

20. Thomas R, Jay JL. Raised intraocular pressure with topical steroids after trabeculectomy. Graefes Arch Clin Exp Ophthalmol 1988;226:337-40.

21. Araujo SV, Spaeth GL, Roth SM, et al. A ten-year follow-up on a prospective, randomized trial of postoperative corticosteroids after trabeculectomy. Ophthalmology 1995;102:1753-9.

22. Shaarawy T, Dada T, Bhartiya S. ISGS textbook of glaucoma surgery. Jaypee Brothers,Medical Publishers Pvt, 2014.

23. Lütjen-Drecoll E, Bárány EH. Functional and electron microscopic changes in the trabecular meshwork remaining after trabeculectomy in cynomolgus monkeys. Invest Ophthalmol 1974;13:511-24.

24. Johnson DH, Matsumoto Y. Schlemm's canal becomes smaller after successful filtration surgery. Arch Ophthalmol 2000;118:1251-6.

25. Overby DR, Clark AF. Animal models of glucocorticoid-induced glaucoma. Exp Eye Res 2015;141:15-22. 